\title{
Contents
}

\section{Part I Preliminaries}

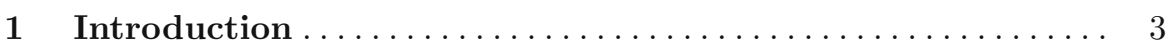

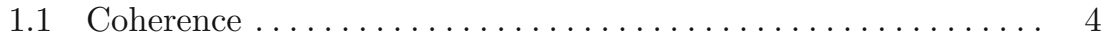

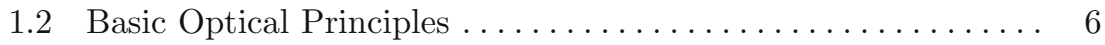

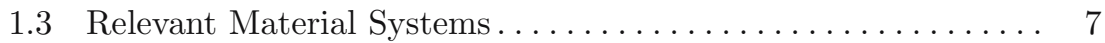

1.4 Related Systems ........................ 8

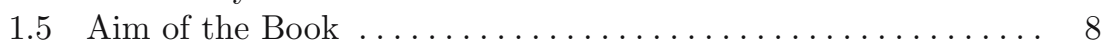

1.6 Necessary Prerequisites. . . . . . . . . . . . . . . . . . 10

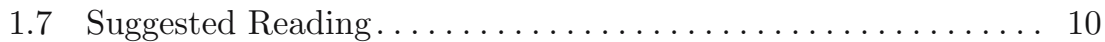

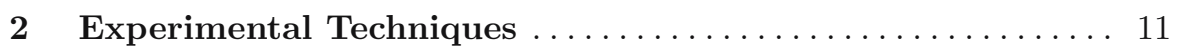

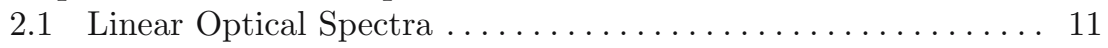

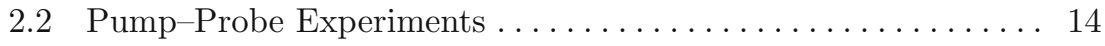

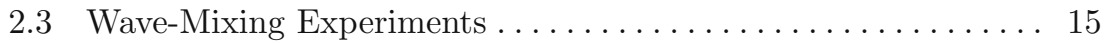

2.4 Transport Phenomena..................... 15

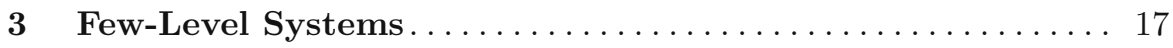

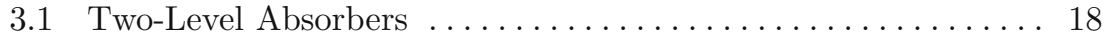

3.2 Three- and More-Level Absorbers . . . . . . . . . . . . . . . . . . 21

3.3 Continua . . . . . . . . . . . . . . . . . . . . . . 21

3.4 Fano Situations .......................... 23

3.5 Suggested Reading......................... 24

4 Coherent Tunneling $\ldots \ldots \ldots \ldots \ldots \ldots \ldots \ldots \ldots \ldots \ldots \ldots$

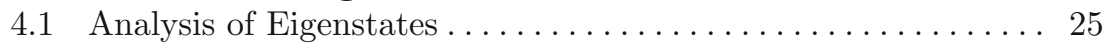

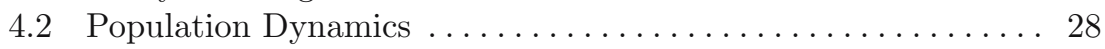

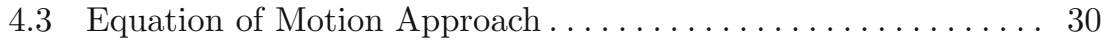

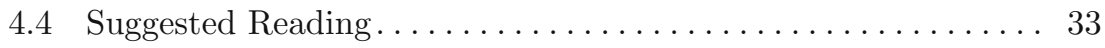




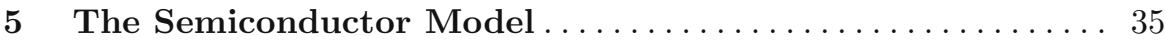

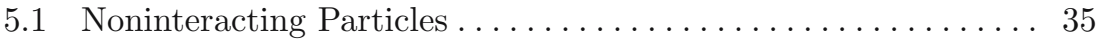

5.1 .1 The $1 \times 1$-Tight-Binding Model ............... 35

5.1 .2 The $2 \times 2$-Tight-Binding Model ............ 36

5.1 .3 The Electron-Hole Picture . . . . . . . . . . . . . . . . 39

5.1 .4 Periodic Boundary Conditions . . . . . . . . . . . 40

5.1 .5 Ordered and Disordered Systems .............. 40

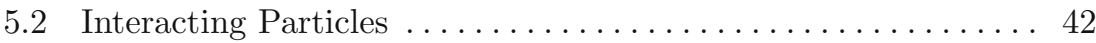

5.3 Electron-Phonon Interaction $\ldots \ldots \ldots \ldots \ldots \ldots \ldots \ldots \ldots \ldots \ldots$

5.4 Electron-Light Interaction . . . . . . . . . . . . . . . . 44

6 Single-Particle Properties . . . . . . . . . . . . . . . . . . . . . . 47

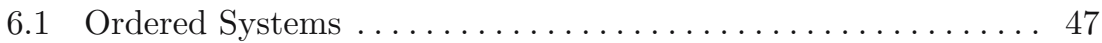

6.1 .1 States.............................. 47

6.1 .2 Eigenvalues ........................ 48

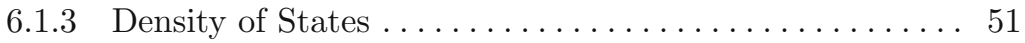

6.1 .4 Optical Spectrum .................... 52

6.1.5 Wave Packets and Propagation .............. 53

6.1.6 Dimerized Systems ... . . . . . . . . . . . . . . . . 55

6.2 Disordered Systems . . . . . . . . . . . . . . . . . . . 55

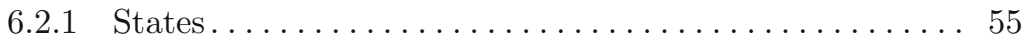

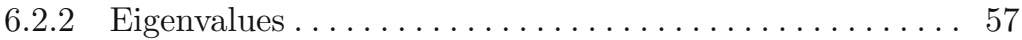

6.2 .3 Density of States . . . . . . . . . . . . . . . 57

6.2 .4 Optical Spectrum .................... 58

6.2.5 Wave Packets and Propagation.............. 58

$7 \quad$ The Equation of Motion Approach ................ 61

7.1 Observables............................ 61

7.2 Noninteracting Particles ....................... 63

7.2.1 The Two-Level Bloch Equations . . . . . . . . . . . . . 63

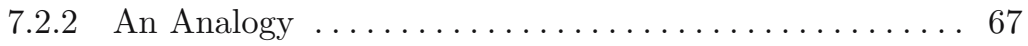

7.2 .3 Dynamics of Optical Excitations . . . . . . . . . . . . . 69

7.2.4 The Few-Level Bloch Equations . . . . . . . . . . . . 73

7.2 .5 Fano Situations . . . . . . . . . . . . . . . . 74

8 Dynamical Equations for Semiconductors . . . . . . . . . . . 75

8.1 Noninteracting Particles . . . . . . . . . . . . . . . . . . . 75

8.2 Interacting Particles . . . . . . . . . . . . . . . . . . 79

8.2.1 Hierarchy of Equations . . . . . . . . . . . . . . . 79

8.2.2 Coherent Dynamics-Controlled Truncation Scheme . . . 80

8.2.3 Dynamical Truncation in the Coherent $\chi^{(3)}$ Limit . . . . 88

8.2.4 Hartree-Fock Approach in the Coherent $\chi^{(3)}$ Limit . . . 92

8.2.5 Dynamical Truncation in the Coherent $\chi^{(5)}$ Limit .... . 93

8.3 Suggested Reading. . . . . . . . . . . . . . . . . . . 98 


\section{Part II Applications I}

$9 \quad$ Linear Optical Response ............................ 101

9.1 Linear Response for Two-Level Systems . . . . . . . . . . . . . . . . 102

9.2 Linear Response for Few-Level Systems . . . . . . . . . . . . . 105

9.2 .1 Continua .............................. 106

9.2.2 Homogeneous versus Inhomogeneous Ensembles . . . . . . . 107

9.3 Linear Response for Fano Systems . . . . . . . . . . . . . . . . . 108

9.3.1 Numerical Results ......................... 109

9.3.2 Analytical Discussion ......................... 114

9.4 Linear Response of the Semiconductor . . . . . . . . . . . . 119

9.4.1 Ordered Semiconductor ...................... 119

9.4 .2 Dimerized Lattice ........................ 124

9.4.3 Disordered Noninteracting Semiconductor ........... 132

9.4.4 Disordered Interacting Semiconductor . . . . . . . . . . . . 134

$9.5 \quad$ Suggested Reading........................... 139

10 Coherent $\chi^{(3)}$ Processes for Level Systems .............. 141

10.1 Coherent $\chi^{(3)}$ Processes for Two-Level Systems. . . . . . . . . . . 142

10.1.1 Pump-Probe Experiments . . . . . . . . . . . . . . . . 142

10.1.2 Coherent Spectral Oscillations . . . . . . . . . . . . . 145

10.1.3 Optical Stark Effect . . . . . . . . . . . . . . . . . . 147

10.1.4 Hole Burning . ........................ 151

10.1.5 Four-Wave-Mixing Experiments ................... 153

10.1.6 Polarization Interference . . . . . . . . . . . . . . 156

10.1.7 Photon Echoes . . . . . . . . . . . . . . . . . . . 157

10.2 Coherent $\chi^{(3)}$ Processes for Few-Level Systems . . . . . . . . . . . 159

10.2.1 Pump-Probe Experiments . . . . . . . . . . . . . . . 160

10.2.2 Four-Wave-Mixing Experiments . . . . . . . . . . . . . 164

10.2.3 Quantum Beats ............................. 164

10.2.4 Dephasing due to Continua .................. 167

10.3 Coherent $\chi^{(3)}$ Processes for Fano Systems ............. 170

10.4 Homogeneous versus Inhomogeneous Ensembles ............ 171

10.5 Suggested Reading. ............................ 174

11 Coherent $\chi^{(3)}$ and $\chi^{(5)}$ Processes

in Ordered Semiconductors . . . . . . . . . . . . . . . . . 177

11.1 Noninteracting Particles . . . . . . . . . . . . . . . . . . . . 178

11.2 Interacting Particles ............................. 178

11.2.1 Pump-Probe Experiments . . . . . . . . . . . . . . . . 180

11.2.2 Four-Wave Mixing . . . . . . . . . . . . . . . . . . . 188

11.3 Suggested Reading................................ 195 
12 Coherent $\chi^{(3)}$ and $\chi^{(5)}$ Processes

in Disordered Semiconductors . . . . . . . . . . . . . . . . . 197

12.1 Noninteracting Particles .......................... 198

12.1.1 Pump-Probe Experiments . . . . . . . . . . . . . . . . . . . . . 198

12.1.2 Four-Wave-Mixing Experiments . . . . . . . . . . . . . . . . 198

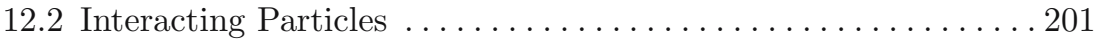

12.2.1 Pump-Probe Experiments ...................... 201

12.2.2 Four-Wave Mixing in the $\chi^{(3)}$ Limit .............. 203

12.2.3 Four-Wave Mixing in the $\chi^{(5)}$ Limit ................211

12.3 Suggested Reading............................. 213

13 Coherent Excitation Spectroscopy ...................215

13.1 Level Systems . . . . . . . . . . . . . . . . . . . . . . . . . . . . . . 215

13.2 Ordered Semiconductors........................ 217

13.3 Disordered Semiconductors . . . . . . . . . . . . . . . . . . . . 218

13.4 Suggested Reading.............................. 219

14 Character of Continuum Transitions . . . . . . . . . . . . . 221

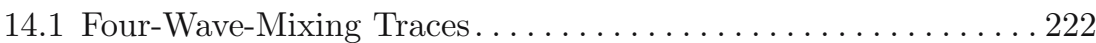

14.2 Coherent Excitation Spectroscopy $\ldots \ldots \ldots \ldots \ldots \ldots \ldots \ldots \ldots \ldots \ldots \ldots$

\section{Part III Applications II}

15 The Semiconductor with Applied Electric Field ........... 229

15.1 Single-Particle Properties . . . . . . . . . . . . . . . . . . . . . . . . . 230

15.1.1 Linear Optical Response ....................238

15.1.2 Coherent Density Dynamics .................... 242

15.1.3 Coherent $\chi^{(3)}$ Processes .......................... . 243

15.1.4 Dynamical Localization ...................... 245

15.2 Interacting System . . . . . . . . . . . . . . . . . . 246

15.2.1 Linear Optical Response ... . . . . . . . . . . . . . . . . . 247

15.2.2 Coherent Density Dynamics ..................... 2 249

15.2.3 Coherent $\chi^{(3)}$ Processes . . . . . . . . . . . . . . . . . . . . 249

15.2.4 Dynamical Localization ...................... 251

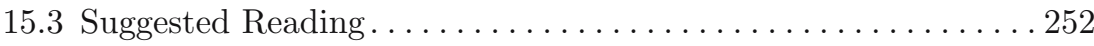

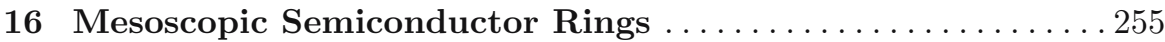

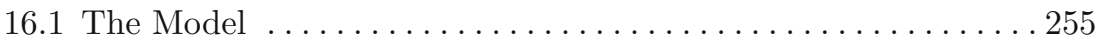

16.1.1 No Magnetic Field......................... 255

16.1.2 Magnetic Field Included . . . . . . . . . . . . . . . . . . . . . . 257

16.2 Single-Particle Properties . . . . . . . . . . . . . . . . . . . . 260

16.2.1 The Single-Particle Spectrum . . . . . . . . . . . . . 260

16.2.2 Persistent Currents ........................261 
16.3 Dynamics of the Electron-Hole Dipole . . . . . . . . . . . 262

16.3.1 Noninteracting Particles . . . . . . . . . . . . . . . . . 262

16.3.2 Interacting Particles . . . . . . . . . . . . . . . 266

16.4 Linear and Nonlinear Magneto-Optics . . . . . . . . . . . . . . . . . 269

16.4.1 Linear Optical Spectra . . . . . . . . . . . . . . . 270

16.4.2 Nonlinear Optical Spectra . . . . . . . . . . . . . . . 272

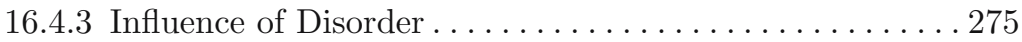

16.5 Suggested Reading. . . . . . . . . . . . . . . . . 277

17 Coherent Density Dynamics

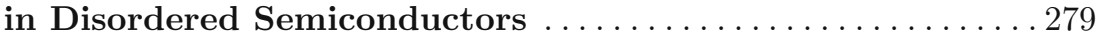

17.1 The Two-Interacting-Particles Problem. . . . . . . . . . . . . 279

17.2 The Semiconductor Two-Interacting-Particles Problem . . . . . . . 280

17.2.1 The Model . . . . . . . . . . . . . . . . . . . 280

17.2.2 Electron-Hole Symmetry . . . . . . . . . . . . . . . 281

17.2.3 Dynamic-Correlation-Induced Delocalization . . . . . . . . 283

17.2.4 Finite-Time Scaling . . . . . . . . . . . . . . . . . 285

17.2.5 Influence of Correlated Versus Anticorrelated

Disorder .......................... 286

17.3 Suggested Reading. . . . . . . . . . . . . . . . . . 290

18 Current Echoes . . . . . . . . . . . . . . . . . . . . . . . . 291

18.1 Current Generation by Coherent Control . . . . . . . . . . . . . . 292

18.2 Current Decay in Disordered Semiconductors . . . . . . . . . . 295

18.3 Equal Electron and Hole Masses . . . . . . . . . . . . . . . . . 297

18.4 Different Electron and Hole Masses . . . . . . . . . . . . . . . . . 297

18.5 Excitation Intensity Dependence . . . . . . . . . . . . . . . . . . 299

18.6 Intraband Dynamics . . . . . . . . . . . . . . . . . . . . 300

18.7 Suggested Reading. . . . . . . . . . . . . . . . . . . 303

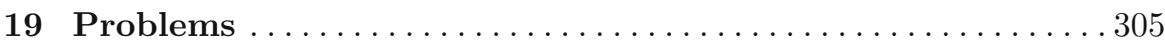

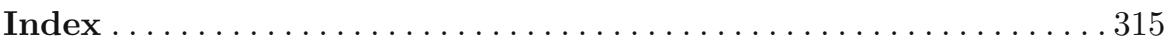

